\title{
RECEIVED
}

JAN 261998

$08 \mathrm{TI}$

\section{H-MODE PEDESTAL CHARACTERISTICS, ELMS, AND ENERGY CONFINEMENT IN ITER SHAPE DISCHARGES ON DIII-D}

by

T.H. OSBORNE, R.J. GROEBNER, L.L. LAO, A.W. LEONARD, R. MAINGI, R.L. MILLER, G.D. PORTER, D.M. THOMAS, and R.E. WALTZ

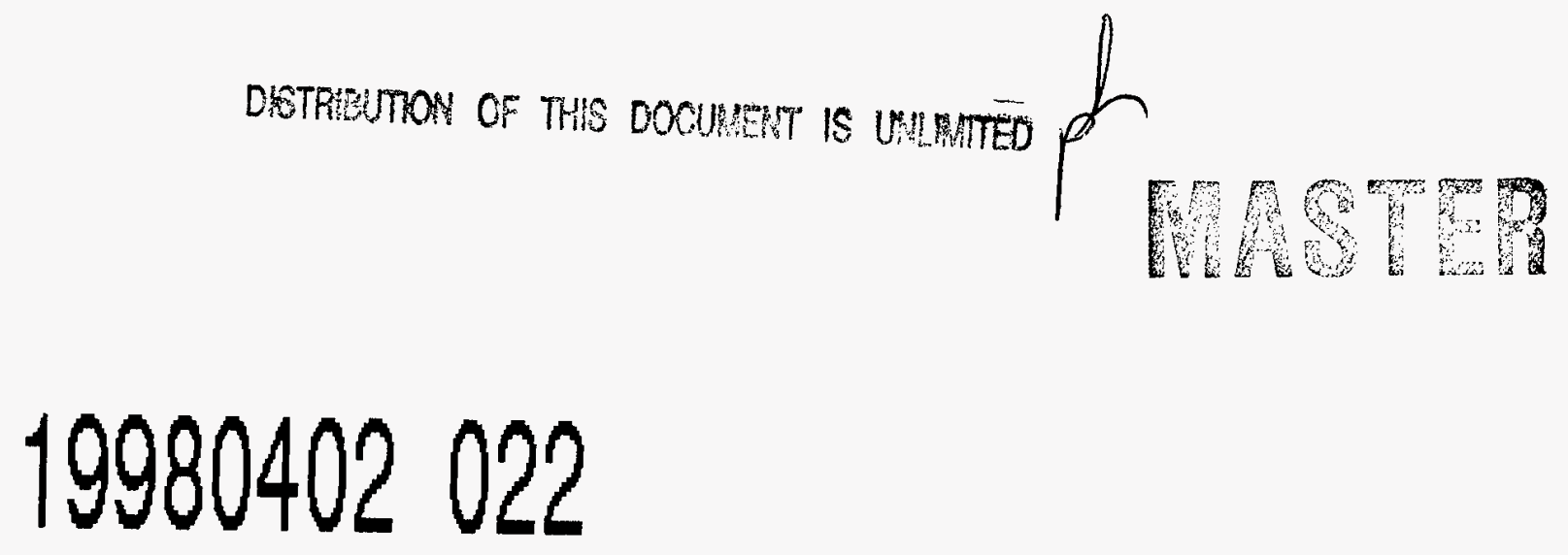

DIC QUALFY IUE EUTED 8

DECEMBER 1997 
GA-A22733

\title{
H-MODE PEDESTAL CHARACTERISTICS, ELMs, AND ENERGY CONFINEMENT IN ITER SHAPE DISCHARGES ON DIII-D
}

\author{
by \\ T.H. OSBORNE, R.J. GROEBNER, L.L. LAO, A.W. LEONARD, R. MAINGI, $†$

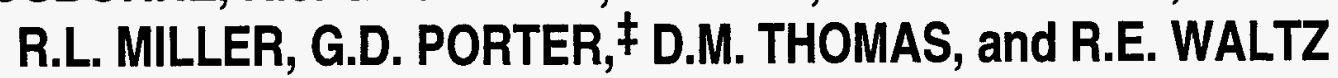 \\ toak Ridge National Laboratory \\ ‡Lawrence Livermore National Laboratory
}

This is a preprint of a paper to be presented at the IAEA Technical Committee Meeting on H-mode Physics, September 22-24, 1997, Kloster-Seeon, Germany and to be published in Special Issue of Plasma Physics \& Controlled Fusion.

\author{
Work supported by \\ the U.S. Department of Energy \\ under Contract Nos. DE-AC03-89ER51114, DE-AC05-960R22464, \\ and W-7405-ENG-48
}

DTIC QUALTYY IATHETUTRD 3

GA PROJECT 3466

DECEMBER 1997 


\section{ABSTRACT}

The $\mathrm{H}$-mode confinement enhancement factor, $\mathrm{H}$, is found to be strongly correlated with the height of the edge pressure pedestal in ITER shape discharges. In discharges with Type I ELMs the pedestal pressure is set by the maximum pressure gradient before the ELM and the width of the $\mathrm{H}$-mode transport barrier. The pressure gradient before Type I ELMs is found to scale as would be expected for a stability limit set by ideal ballooning modes, but with values significantly in excess of that predicted by stability code calculations. The width of the H-mode transport barrier is found to scale equally well with pedestal $\rho_{\mathrm{POL}}^{2 / 3}$ or $\beta_{\mathrm{POL}}^{1 / 2}$. The improved $\mathrm{H}$ value in high $\beta_{\text {POL }}$ discharges may be due to a larger edge pressure gradient and wider $\mathrm{H}$-mode transport barrier consistent with their higher edge ballooning mode limit. Deuterium puffing is found to reduce $\mathrm{H}$ consistent with the smaller pedestal pressure which results from the reduced barrier width and critical pressure gradient. Type I ELM energy loss is found to be proportional to the change in the pedestal energy. 


\section{INTRODUCTION}

A capability to predict the characteristics of the H-mode edge pedestal in ITER and other future devices is important in at least two areas. Stiff ion temperature gradient, ITG,-mode turbulent transport models $[2,3]$ indicate that the core transport coefficients depend strongly on the plasma edge conditions. This result is supported by experiments presented in this paper and elsewhere $[4,8]$. The more pessimistic models predict that ITER will reach its design goal of $\mathrm{Q}=$ 15 only with edge ion temperature above $4 \mathrm{keV}$. The $\mathrm{H}$-mode pedestal characteristics are also important in their connection to ELM stability and energy loss. The current ITER divertor design can tolerate $1 \mathrm{MJ} / \mathrm{m}^{2} / \mathrm{ELM}$ or $10 \mathrm{MJ} / \mathrm{ELM}$ (a loss of $1 \%$ of the plasma stored energy per ELM at ignition) to the divertor plates if the energy arrives on a time scale consistent with present experiments. In this paper we describe experimental results from DIII-D pertaining to the scaling of the height of the H-mode pressure pedestal and ELM energy loss. These experiments primarily employed discharges with ITER cross sectional shape and aspect ratio $\left(\mathrm{L}_{\mathrm{DIII}-\mathrm{D}} / \mathrm{L}_{\mathrm{ITER}}=0.2\right.$, where $\mathrm{L}$ is the length scale). These were gas puff fueled discharges in an open divertor configuration, with the ion $\nabla \mathrm{B}$ drift toward the $\mathrm{X}$-point. A database of some 75 discharges was assembled with data from: 1) Thomson scattering measurements of electron density and temperature profiles, 2 ) in a few cases charge exchange recombination measurements of ion temperaute and rotation, 3) EFIT MHD equilibrium parameters, 4) results of BALOO [5] ballooning mode stability calculations, 5) ELM frequency, energy loss, and divertor effects, 6) MHD fluctuation levels, 7) other parameters such as divertor neutral pressure and heating power. In all, roughly 250 parameters for 10,000 data points were included, for $\mathrm{L}$-mode, various ELM types, and ELM free discharges. 


\section{H-MODE PEDESTAL HEIGHT AND ENERGY CONFINEMENT}

A regression analysis including all ELM classes, ELM free, and L-mode on the data base described in Section 1 gave a scaling, H-ITER93-H $\propto\left(\mathrm{T}_{\mathrm{e}}^{\mathrm{PED}}\right)^{0.55}\left(\mathrm{n}_{\mathrm{e}}^{\mathrm{PED}}\right)^{0.58} / \mathrm{B}_{\mathrm{T}}^{0.93}$, where $\mathrm{H}$-ITER93- $\mathrm{H}$ is the confinement time divided by the scaling relation derived for $\mathrm{H}$-mode with Type I ELMs for ITER, and PED refers to the values at the top of the H-mode pedestal. We could not separately determine a density and poloidal field dependence because of the correlation between these parameters in the data set used so far. A fit using temperature alone and including poloidal field gave roughly a factor of 5 higher $\chi^{2}$, suggesting that pedestal electron pressure may be a more important parameter in determining $H$. For a data set at fixed $I_{P}$ and $B_{T}$, fits of $H$ to $\mathrm{n}_{\mathrm{e}}^{\mathrm{PED}}$ or $\mathrm{T}_{\mathrm{e}}^{\mathrm{PED}}$ separately gave a factor of 4 larger $\chi^{2}$ than a fit to $\mathrm{P}_{\mathrm{e}}^{\mathrm{PED}}$. Type I and III ELMs [1] appeared in distinct regions of $\mathrm{n}_{\mathrm{e}}^{\mathrm{PED}}, \mathrm{T}_{\mathrm{e}}^{\mathrm{PED}}$ space [8], with type I ELMs occurring roughly along a constant $\mathrm{P}_{\mathrm{e}}^{\mathrm{PED}}$ contour. Variation in pedestal pressure within and between ELM types is correlated with confinement variations. Since the width of the H-mode transport barrier is expected to be set by turbulence suppression physics [6], and the magnitude of the gradient may be limited by the ELM instability [7], we treat the scaling of these two quantities separately in the sections that follow. 


\section{H-MODE TRANSPORT BARRIER WIDTH SCALING}

The region of the $\mathrm{H}$-mode transport barrier is assumed to coincide with the high electron pressure gradient region near the separatrix as determined from Thomson scattering measurements of the electron temperature and density. In a few cases where comparisons have been made, we find that the width of the edge steep gradient region for the ion pressure matches that of the electrons. The scaling of the $\mathrm{H}$-mode transport barrier width has been determined only for the Type I ELM regime. Only $\mathrm{P}_{e}^{\mathrm{PED}}, \mathrm{T}_{\mathrm{e}}^{\mathrm{PED}}$, and $\mathrm{BPOL}_{\mathrm{PO}}$ had significant correlation with the transport barrier width. In the regression analysis the width of the steep gradient region on the outboard midplane was fit equally well to the pedestal pressure, $\delta / \mathrm{R} \propto\left(\beta_{\mathrm{POL}}^{\mathrm{PED}}\right)^{0.4}$, or pedestal temperature, $\delta / \mathrm{R} \propto\left(\rho_{\mathrm{POL}}^{\mathrm{PED}} / \mathrm{R}\right)^{0.66}$, where only electron parameters are used in $\beta_{\mathrm{POL}}^{\mathrm{PED}}$ and $\rho_{\mathrm{POL}}^{\mathrm{PED}}$

In discharges with divertor pumping, in which $T_{e}^{P E D}$ increased at constant $P_{e}^{P E D}$ (Fig. 1.), linear $\rho \mathrm{PED}$ dependence appears to be ruled out within the uncertainty of the $\delta$ measurement, however weaker $\rho$ POL dependencies such as obtained in the scalings above may still be possible.

For Type III ELMs the barrier width is 20 to $50 \%$ larger than for Type I even though $\mathrm{P}_{\mathrm{e}}^{\mathrm{PED}}$ and $\mathrm{T}_{\mathrm{e}}^{\mathrm{PED}}$ are typically significantly smaller. 

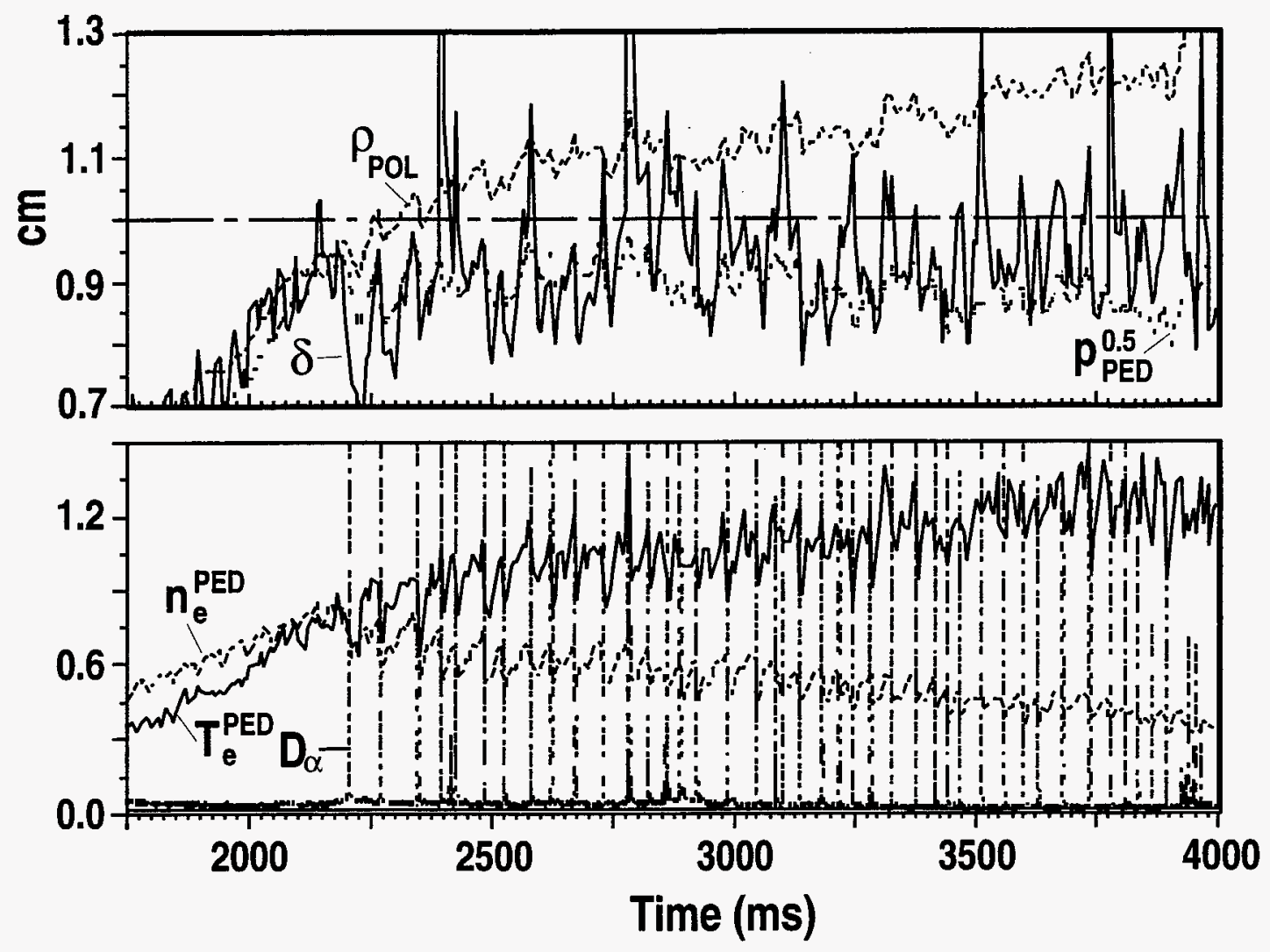

Fig. 1. Time evolution of $\mathbf{H}$-mode transport barrier width ( $\delta$-- Solid), poloidal gyro radius ( $\rho_{\mathrm{POL}}-$ - Dash), and width scaling $\left(\left(\mathrm{p}_{e}{ }^{\text {PED }}\right)^{1 / 2}-\right.$ Dot $)$ along with electron temperature $\left(T_{e}-\right.$ Solid $)$, electron density $\left(n_{e}\left(10^{20} / m^{3}\right)\right.$ -- Dash), and $D_{\alpha}$ (dot) for a discharge with divertor pumping showing that $\delta$ remains constant while $\rho_{\text {POL }}$ increases of $\delta$ with the pressure scaling. 


\section{EDGE PRESSURE GRADIENT AND BALLOONING STABILITY}

In these experiments we find that the electron pressure gradient before a type I ELM, scales as would be expected for an ideal ballooning mode limit, with $\alpha_{\mathrm{MHD}}=2 \mu_{0}\left(\mathrm{dp}_{\mathrm{e}} / \mathrm{d} \psi\right)(\mathrm{dV} / \mathrm{d} \psi)\left[\mathrm{V} /\left(2 \pi^{2} \mathrm{R}\right)\right]^{1 / 2} / 4 \pi^{2}$, relatively independent of $q[8]$. We can estimate the edge temperature in ITER by assuming Type I ELMs will occur at the same $\alpha$ as in DIII-D at ITER $q$. Scaling from DIII-D discharges, $\delta / R \propto\left(\rho_{\mathrm{POL}} / \mathrm{R}\right)^{2 / 3}$ gives $\mathrm{T}_{\mathrm{e}}^{\mathrm{PED}} \approx 1 \mathrm{keV}$ for ITER, and for $\delta / \mathrm{R} \propto\left(\beta_{\mathrm{POL}}^{\mathrm{PED}}\right)^{1 / 2}$, gives $\mathrm{T}_{\mathrm{e}}^{\mathrm{PED}} \approx 5 \mathrm{keV}$ for ITER.

Although $\alpha_{\text {MHD }}$ does not vary with $q$, the pressure gradient on the outboard midplane increases with heating power in high $q\left(q_{95}=6.5\right)$ discharges. This increase is correlated with $\beta_{\text {POL increasing, and is consistent with the increase in computed ballooning mode critical }}$ pressure gradient which results from BPoL increasing on the outboard midplane. This increase in pressure gradient and the associated increase in transport barrier width, as expected from the scaling given in Section 3, may result in the high pedestal pressure observed in these discharges. The $\mathrm{H}$ factor is also observed to increase with $\beta_{\mathrm{POL}}$ in these discharges (Fig. 2), consistent with the scaling of $\mathrm{H}$ with $\mathrm{P}_{\mathrm{e}}^{\mathrm{PED}}$ given in Section 2 . The increase in $\mathrm{H}$ is significantly larger than would be expected from the pedestal energy increase alone.

Although the edge pressure gradient scales as would be expected for ideal ballooning, the value of the electron pressure gradient is, however, approximately equal to total pressure gradient limit as predicted by stability code calculations using the BALOO code [5] with current density profiles determined from MHD equilibria fit to external magnetics only. Since the measured ion pressure gradient equals or exceeds the electron pressure gradient, this means the experimentally measured pressure is at least twice the calculated value. Stability analysis results including the current density expected to be associated as bootstrap current with the edge pressure gradient indicate that local regions of second stable access can be created in regions of high pressure gradient [9]. 


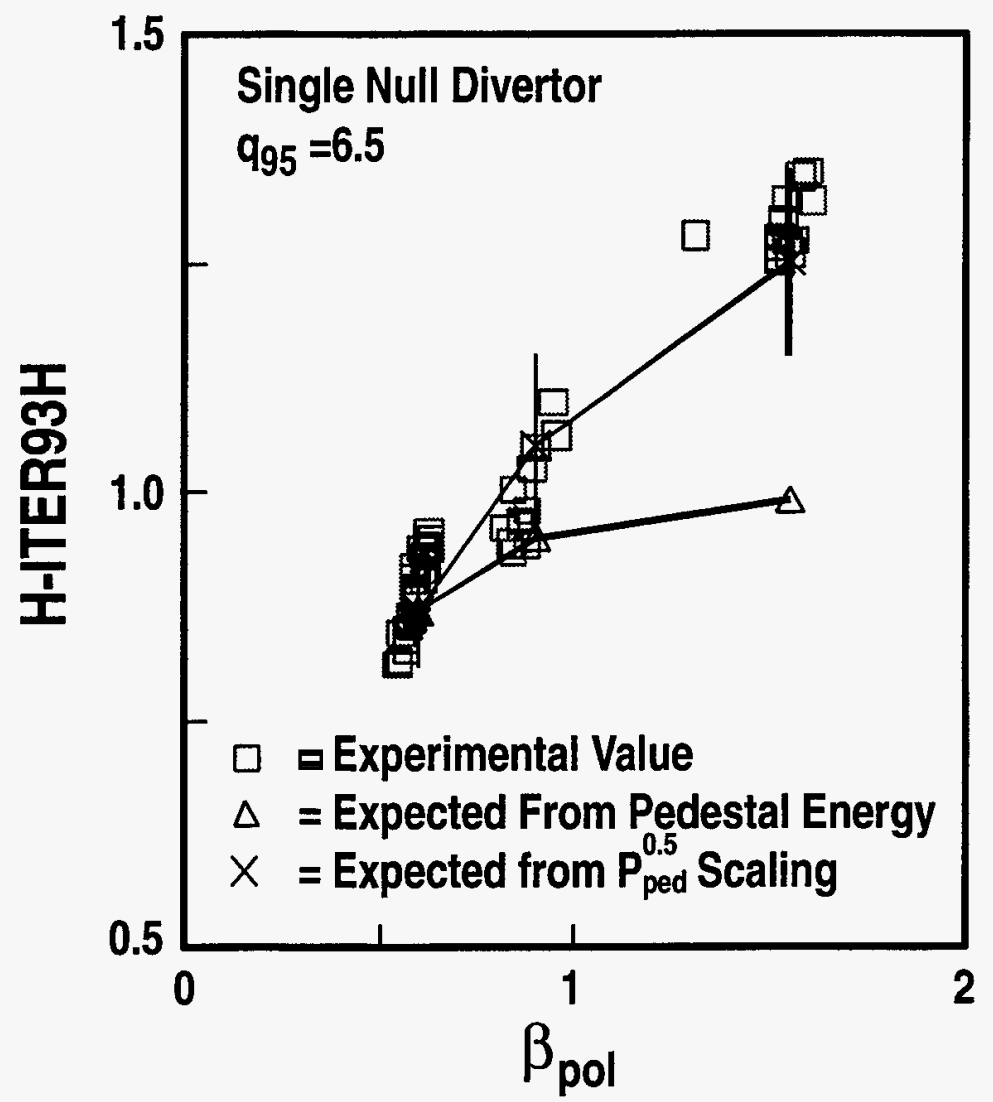

Fig. 2. Confinement enhancement factor, $\mathrm{H}$, relative to ITER93 $\mathrm{H}$-mode scaling increases with $\beta_{\mathrm{POL}}$ in agreement with pedestal pressure scaling and significantly above what would be expected from the incense in pedestal energy alone. 


\section{TYPE I ELM ENERGY LOSS}

The energy lost from the plasma core during Type I ELMs was determined from the time history of the stored energy obtained from MHD equilibria. This method was found to be in agreement, within experimental uncertainties, with the energy deposited in the divertor determined from IR camera measurements [10]. A regression analysis of this data [8] gives, $\Delta \mathrm{E}_{\mathrm{ELM}} / \mathrm{E}_{\mathrm{TOTAL}} \propto(\mathrm{P} / \mathrm{S})^{-0.4} \mathrm{~B}^{-0.3}$ for the fraction of stored energy lost per ELM, where $\mathrm{P}$ is the total input power and $\mathrm{S}$ is the plasma surface area. This relation gives $\Delta \mathrm{E}_{\mathrm{ELM}} \cong 26 \mathrm{MJ}$ for ITER. The ELM energy loss was found to be correlated with the energy change in the H-mode pedestal $\Delta \mathrm{E}_{\mathrm{ELM}} \cong \Delta \mathrm{P}_{\mathrm{e}}^{\mathrm{PED}} \mathrm{V} / 2$ and, to a lesser degree, to the pedestal energy before the ELM, $\Delta \mathrm{E}_{\mathrm{ELM}} \cong$ $\mathrm{P}_{\mathrm{e}}^{\mathrm{PED}}$ V/3.5 (Fig. 3). The weaker correlation of $\Delta \mathrm{E}_{\mathrm{ELM}}$ with the pedestal energy results from a variation in the pedestal energy after the ELM, the scaling of which has not yet been investegated. The later relation implies $\Delta \mathrm{E}_{\mathrm{ELM}} \cong 8 \mathrm{~T}^{\mathrm{PED}}(\mathrm{MJ} / \mathrm{keV})$. From the ITER $\mathrm{T}^{\mathrm{PED}}$ estimates based on the $\beta_{\mathrm{POL}}^{\mathrm{PED}}$ and $\rho_{\mathrm{POL}}^{\mathrm{PED}}$ scaling given in the previous section, this gives $\Delta \mathrm{E}_{\mathrm{ELM}}$ $=40$ and $8 \mathrm{MJ}$ respectively. The time scale for the ELM energy loss is typically less than $1 \mathrm{~ms}$.

Discharges near the $\mathrm{H}$-mode threshold power develop compound ELMs [1] at reduced frequency with deuterium puffing while discharges far from threshold show increased ELM frequency. In either case the average ELM power loss is found to remain relatively fixed with deuterium puffing to a certainty sufficient to suggest that the reduced $\mathbf{H}$ is not the result of increased ELM loss power. In contrast, $\triangle E_{E L M}$ is relatively independent of heating power while the frequency and hence average ELM power loss increases with heating power. 


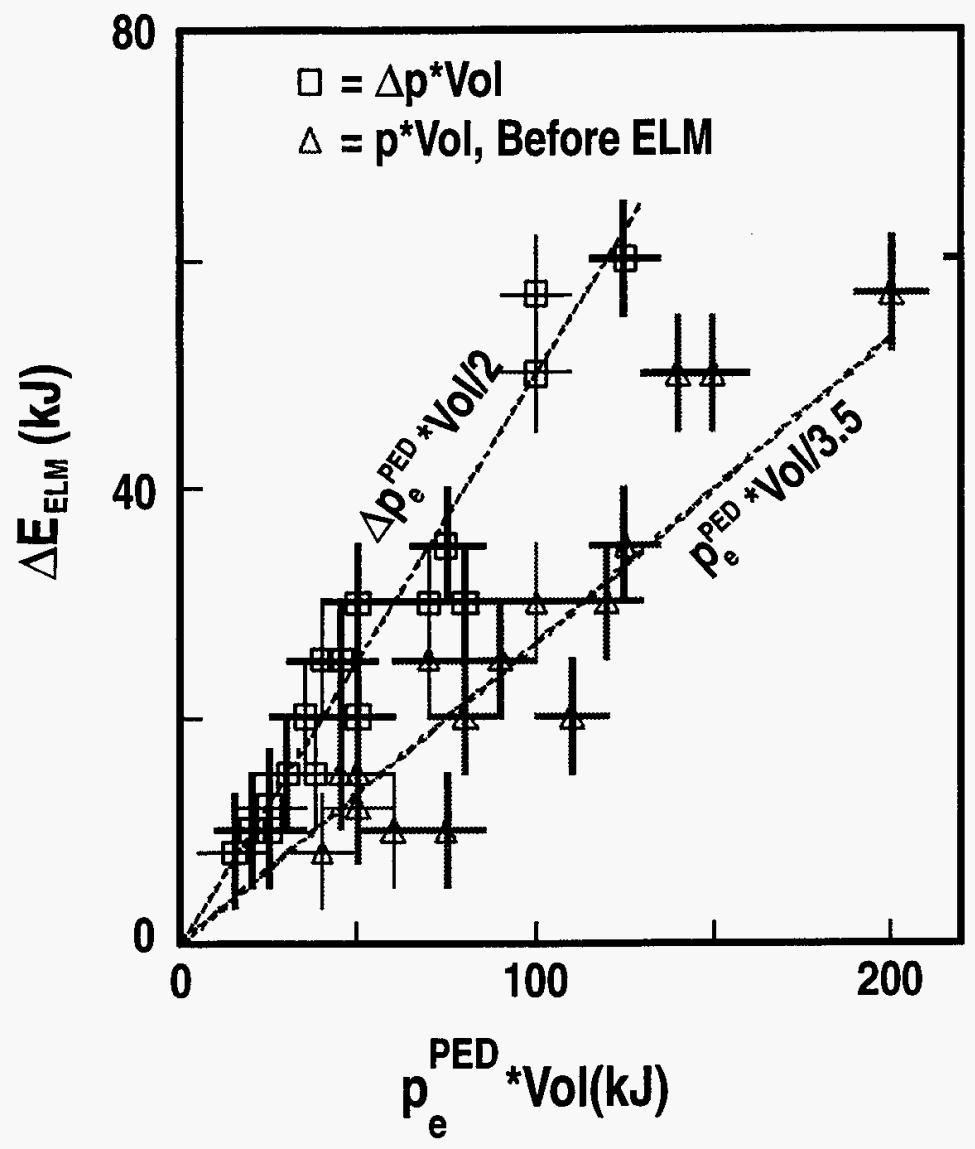

Fig. 3. Type I ELM energy loss, $\Delta \mathrm{E}_{\mathrm{ELM}}$, agrees well with half the change in the electron pedestal energy, $\Delta \mathrm{p}_{\mathrm{e}}{ }^{\mathrm{PED}} \mathrm{V} / 2$, where $\mathrm{V}$ is the total plasma volume, and to a lesser degree with $30 \%$ of the electron pedestal energy, $\mathrm{p}_{\mathrm{e}}{ }^{\mathrm{PED}} \mathrm{V} / 3.5$. 


\section{SUMMARY}

The energy confinement quality is correlated with the height of the $\mathrm{H}$-mode pedestal over a range of plasm conditions in DIII-D as might be expected from stiff profile transport models. An understanding of the factors which control the pedestal parameters then may provide a means for predicting the confinement quality in future devices. In the type I ELM regime on DIII-D the width of the $\mathrm{H}$-mode transport barrier is correlated to a similar degree with the edge electron pressure and temperature, although the results of divertor pumping experiments suggests that pressure is the more important parameter. Changes in the neutral particle source, which will be studied more fully in future work, might also play a role in the $\mathrm{H}$-mode pedestal parameters in the discharges with gas puffing or divertor pumping. Variations in the profile of the particle source can effect the radial electric field profile near the edge. Neutrals can also produce a drag on plasma rotation or enhance ion orbit loss current. The type I ELM energy loss is found to be proportional to the change in the $\mathrm{H}$-mode pedestal energy, and, to a lesser degree, correlated with the pedestal energy before the ELM. Scaling from the DIII-D results to ITER give pedestal temperatures in the range of 1 to $5 \mathrm{keV}$ and ELM energy loss in the range of 8 to $40 \mathrm{MJ}$ with the later larger values in each case the result of scaling the barrier width with pressure. It is clear that gas puffing in Type 1 ELM discharges can reduce the ELM energy loss; however, so far we have only observed cases in which this effect is accompanied with reduced pedestal height and confinement quality. 


\section{REFERENCES}

[1] H. Zohm, Plasma Phys. Contr. Fusion 38, 105 (1996).

[2] M. Kotschenreuter, et. al., Proc. 16th International Conf. on Plasma Phys. and Controlled Nucl. Fusion Research, Montreal, Canada, October 1996, IAEA-F1-CN-64/D1-5.

[3] R.E. Waltz, et. al., Proc. 16th International Conf. on Plasma Phys. and Controlled Nucl. Fusion Research, Montreal, Canada, October 1996, IAEA-F1-CN-64/D1-6.

[4] A. Hubbard, et. al., Proc. 16th International Conf. on Plasma Phys. and Controlled Nucl. Fusion Research, Montreal, Canada, October 1996, IAEA-F1-CN-64/D1-6.

[5] R.L. Miller, et. al., Physics of Plasmas, 4, 1062 (1997).

[6] K.H. Burrell, et. al., Plasma Phys. and Contr. Fusion, 34, 1859 (1992).

[7] P. Gohil, et. al., Phys. Rev. Lett., 61, 1603 (1988).

[8] T.H. Osborne, et al., 1997 Proc. Twenty-Fourth Euro. Conf. on Controlled Fusion and Plasma Physics, Berchtesgaden, Germany (European Physical Society, to be published).

[9] R.L. Miller, et. al., Plasma Phys. and Contr. Fusion, (to be published 1997).

[10] A. Leonard, et al., J. Nucl. Mat. 241-243,, 628 (1997). 


\section{ACKNOWLEDGMENT}

Work supported by the U.S. Department of Energy under Contracts DE-AC03-89ER51114, DE-AC05-96OR22464, and W-7405-ENG-48. 
M98001905

|||||||||||||||||||||||||||||||||||||||||||||||

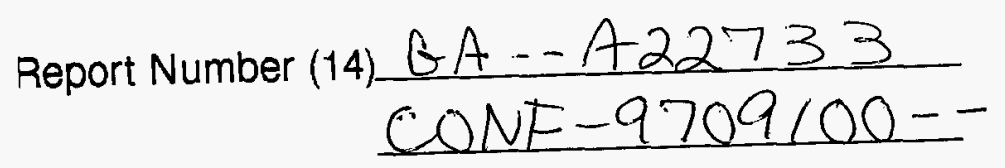

subl. Date (11) 199712

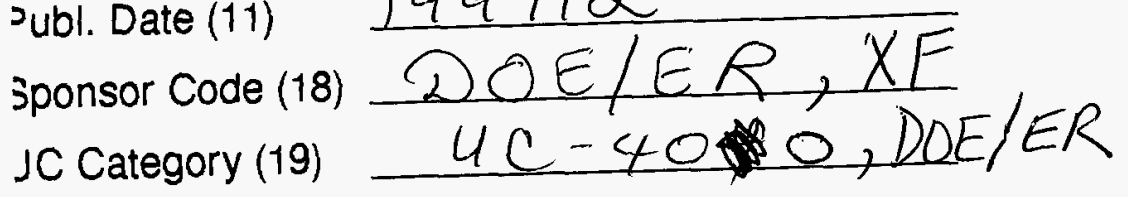

DOE 